EPJ Web of Conferences 52, 01007 (2013)

DOI: $10.1051 /$ epjconf/20135201007

C) Owned by the authors, published by EDP Sciences, 2013

\title{
The results from LHCf and future prospects
}

\author{
Yoshitaka Itow ${ }^{1,2, a}$ for the LHCf collaboration \\ ${ }^{1}$ Solar-Terrestrial Environment Laboratory, Nagoya University \\ ${ }^{2}$ Kobayashi Maskawa Institute for the Origin of particles and the Universe
}

\begin{abstract}
Precise understanding of hadronic interactions at very high energy is essentially important for observations of ultra high energy cosmic rays by extensive air showers. The LHCf experiment provides new data for very forward particle productions produced by $\mathrm{LHC} 7 \mathrm{TeV}$ and $0.9 \mathrm{TeV} p-p$ collisions. These data relevant to air showers development will improve current uncertainty in observation of ultra high energy cosmic rays. Recent LHCf data and future prospects are over-viewed.
\end{abstract}

\section{Introduction}

About 100 years ago, observation of cosmic rays was the main method to study elementary particles and their interactions. After accelerator experiments took over the main activity of study in particles and nuclei, high energy cosmic ray gave still a unique opportunity to explore unknown interactions at such high energy that accelerators could not reach. Also very high energy interactions become important from the view point of implication of data from extensive air shower experiments to observe ultra high energy cosmic rays (UHECRs).

Recently two air shower experiments, Pierre-Auger Observatory (PAO) and Telescope Array (TA) have carried out observation of UHECRs above the $10^{18} \mathrm{eV}$ region. They have revealed existence of "cut-off" feature of energy spectra [1] [2]. These facts are naturally considered as an existence of the GZK cut off [3]. However chemical composition of UHECRs has not yet consistently determined. Chemical composition of high energy cosmic rays is usually determined by shower max height $\left(X_{\max }\right)$. The implication of the $X_{\max }$ distributions of PAO and of TA are not consistent with each others [1] [2]. Experimental situation is still controversial.

$X_{\max }$ depends not only on chemical composition but also on hadronic interactions as for following points; 1 ) inelastic cross sections, 2) forward energy spectra of secondary particles, 3) inelasticity of collisions. These parameters should be determined independently by accelerator experiments.

A proton collider machine with the CM energy $\sqrt{s}=$ $2 E_{\text {beam }}$ can provide the equivalent collision energy for interaction of a UHECR proton of incident energy $E_{C R}$ as ; $E_{C R}=2 E_{\text {beam }}^{2} / M_{p}$, where $M_{p}$ is the proton mass. Thus hadron collider experiments can provide interaction data at very high energy comparable to that of UHECRs.
Inelastic cross sections $\sigma_{\text {inel }}$ have been preciously measured at several collider collider experiments such as ISR, $\mathrm{S} p \bar{p} \mathrm{~S}$, Tevatron and LHC. For example, $p$ - $p$ collisions at $\sqrt{s}=14 \mathrm{TeV}$ correspond to interactions of cosmic rays of $10^{17} \mathrm{eV}$ which is just a few order smaller than the GZK energy region. Cosmic rays of which collision energy is equivalent to that of $\mathrm{LHC}$ is also covered by the existing KASCADE-GRANDE [4] or low energy extension plans of PAO and TA, i.e., HEAT [5] and TALE [6], respectively. Thus $10^{17} \mathrm{eV}$ is interesting energy region where both air showers and LHC collision data can be directly compared. There interesting comparison in particle productions have been already made between LHC data and prediction by the existing cosmic ray interaction models such as SYBILL [7], DPMJET3 [9], QGSJET-II [8], and EPOS [10]. It is interesting that the cosmic ray interaction models describe LHC data even better than existing Pythia-based models. However LHC data are mostly limited in the central rapidity regions $|\eta|<\sim 4$ covered by the main central detectors. Only the TOTEM T2 telescope [11] or CMS CASTOR [12] can access the forward rapidity region upto $|\eta| \sim 6$. Figure 1 shows the energy flow distribution as a function of pseudorapidity $\eta$. The dominant part of collision energy is carried by very forward particles produced at $|\eta| \sim 8$ where Zero Degree Calorimeters (ZDCs) only cover. Precise electro-magnetic (EM) component must be measured at this region to verify air shower development where $97 \%$ of the primary energy is released by EM cascade. The LHCf experiment aims to measure neutral particle production at 0 degree of LHC collisions mainly focusing on EM component such as gamma-rays or neutral pions. Detail description of the detectors and calibration can be found elsewhere [13, 14] There are no other such measurements planed to meausure very forward energy spectra of electromagnetic component, though this quantity is very relevant to shower develoment [15]. There were two independent detectors, Arm1 and Arm2, installed in the $10-\mathrm{cm}$ wide experimental slot

\footnotetext{
ae-mail: itow@stelab.nagoya-u.ac.jp
} 

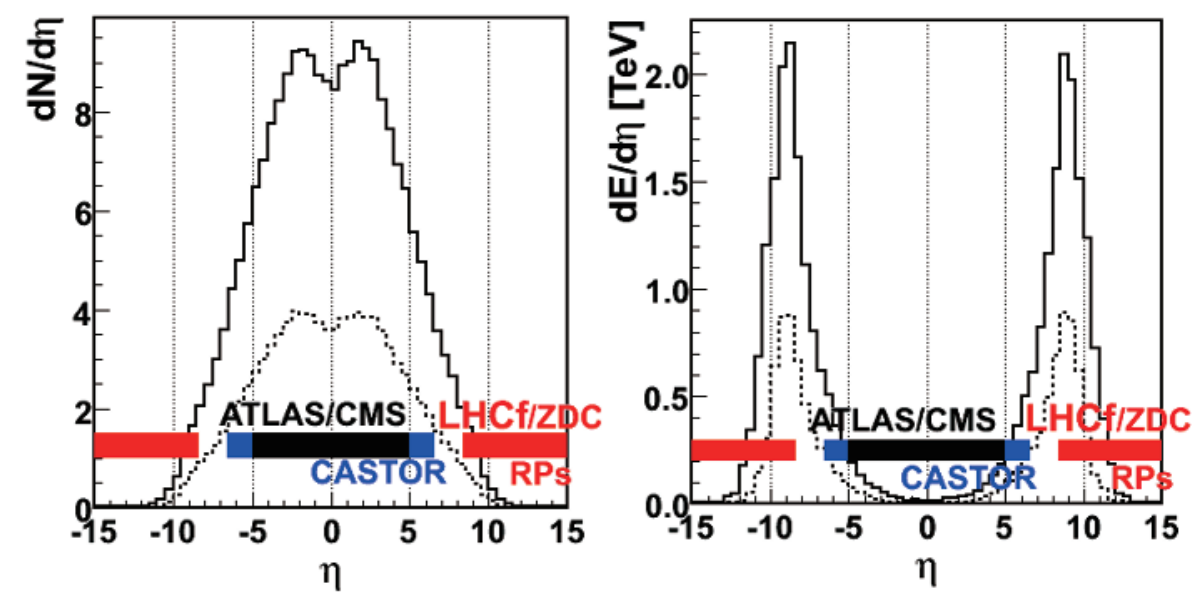

Figure 1. The multiplicity (left) and the energy flow (right) of secondary particles as a function pseudorapidity at $7 \mathrm{TeV} p$ - $p$ collisions. Also shown typical pseudorapidity coverage of existing LHC detectors.

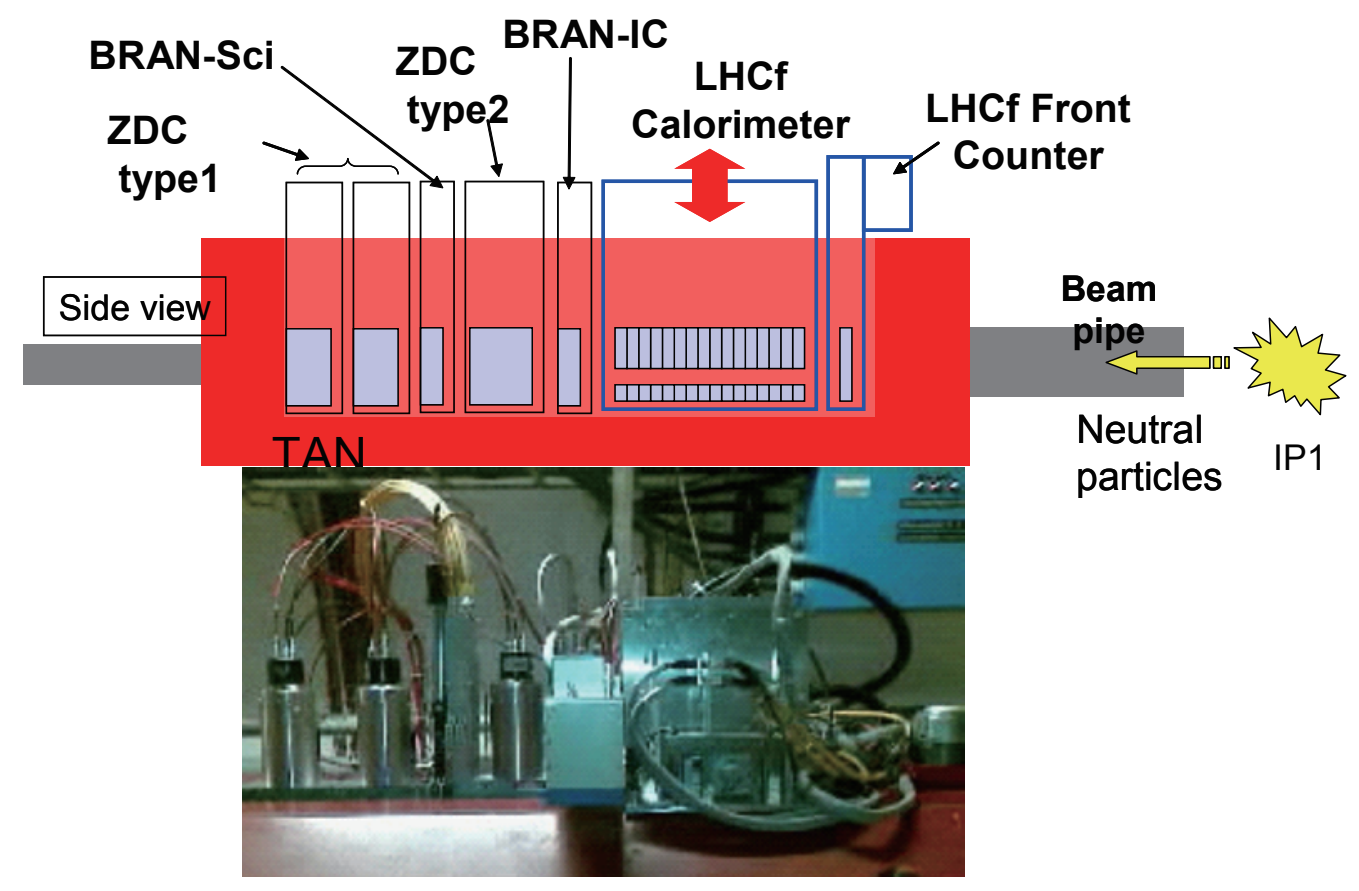

Figure 2. The top figure shows the schematic view of experimental setup of LHCf detectors as well as other detectors in TAN. Here a side view of detector layout is shown. Also shown at the bottom a photo of side view for the TAN region. 
in TAN located $\pm 140 \mathrm{~m}$ from the interaction point IP1. At this location only neutral particles travelling through the beam pipes can be detected, because all the charged particles are swept away by the D1 dipole magnets between the two locations. Figure 2 shows schematic view of experimental setup as well as other detectors in TAN. Each Arm1 or Arm2 detector was installed in the most upstream slot in TAN. Each Arm1 or Arm2 detector could change vertical position in order to change the rapidity coverage. In front of each detector there was a thin plastic scintillation counter, Front Counter (FC). It was used to determine luminosity calibrated by using Van der Meer scan [16]. Downstream the detector, the BRAN luminosity monitor was installed followed by the hadron shower modules of ATLAS Zero Degree Calorimeter (ZDC).

Figure 3 shows schematic view of the Arm1 and Arm2 detectors. Each LHCf Arm1 or Arm2 detector consisted of a small and a large sampling calorimeters (towers) with 16 layers of plastic scintillators interleaved with a tungsten plate of 2 radiation lengths. In total it had $44 \mathrm{X}_{0}$ radiation lengths and 1.6 interaction lengths. The dimensions of calorimeters in Arm1 (Arm2) were $2 \mathrm{~cm} \times 2 \mathrm{~cm}$ ( $2.5 \mathrm{~cm} \times 2.5 \mathrm{~cm})$ and $4 \mathrm{~cm} \times 4 \mathrm{~cm}(3.5 \mathrm{~cm} \times 3.5 \mathrm{~cm})$ for small and large calorimeters, respectively. Each detector had 4 layers of position sensitive layers using $1 \mathrm{~mm}$ plastic scintillating fiber (Arm1) or Si strip detectors (Arm2). Typical position resolutions for Arm1 and Arm2 were $170 \mu \mathrm{m}$ and $40 \mu \mathrm{m}$, respectively. Even with such small calorimeter aperture, energy resolution for EM showers was achieved to be $5 \%$ by correcting shower leakage effect with reconstructed incident position information. Reconstructed incident position was also used to obtain transverse momenta of the particle. Invariant masses of two particles, for example 2 gamma-rays from a $\pi^{0}$ decay, can be reconstructed from the measurements of two towers in the detector. Absolute energy scale was checked with the $\pi^{0}$ invariant mass distribution as well as with the SPS test beam calibrations [17].

In 2010, data taking for $p$ - $p$ collisions at $\sqrt{s}=7 \mathrm{TeV}$ and $0.9 \mathrm{TeV}$ was carried out in the machine commissioning phase with luminosity less than $\sim 10^{30} \mathrm{~cm}^{2} \mathrm{~s}^{-1}$. Before high luminosity $\left(>10^{31} \mathrm{~cm}^{2} \mathrm{~s}^{-1}\right)$ operation at LHC started, the detectors were removed in July 2010. Then Arm2 was installed again for $\mathrm{p}-\mathrm{Pb}$ collisions in 2012 winter. After successful data taking of $\mathrm{p}-\mathrm{Pb}$ collisions, the detector was removed again and disassembled for detector upgrade work.

\section{Results from the LHCf experiment}

\subsection{Single gamma-ray incident sample}

The data used for the single gamma-ray incident sample in $7 \mathrm{TeV} p$ - $p$ collisions was taken on 15 May 2010 with typical luminosity $L \sim 6 \times 10^{28}$. Integrated luminosity corresponds to $0.68 \mathrm{nb}^{-1}\left(0.53 \mathrm{nb}^{-1}\right)$ for Arm1 (Arm2). Analysis of calorimeter data were carried out by following methods. For the first, shower energy was estimated from sum of scintillator layer signals with the correction

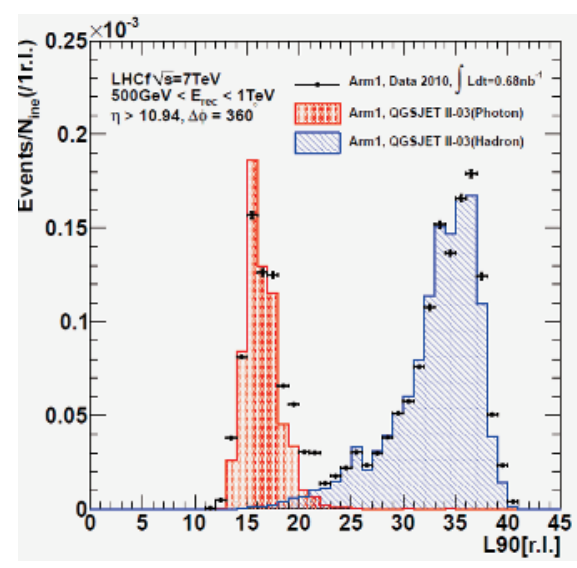

Figure 4. The "L90\%" distribution for the $7 \mathrm{TeV}$ data [19]. The left and the right peaks correspond to EM showers and hadronic showers, respectively. Hatched histograms show the template distributions for single gamma-ray incident (left, hatched-red) and for single hadron incident (right, hatched-blue) events generated with DPMJET3.

of shower leakage effect estimated from the shower incident position. Absolute energy scale was determined by pre-calibration by electron beams at the CERN SPS H4 beam-line and also checked by the invariant mass peaks of $\pi^{0}$ 's reconstructed from $\gamma$-pair samples. Difference between two methods were taken into account as systematic errors of energy scale as $(-9.8 \%,+1.8 \%)$ for Arm1 and $(-6.6 \%,+2.2 \%)$ for Arm2. Single-incident events were selected by using information from position sensitive layers. Then the "L90\%", the radiation lengths where $90 \%$ of visible shower energy is contained, was calculated to discriminate EM showers from hadronic showers. The Fig 4 shows the "L90\%" distribution for $7 \mathrm{TeV} p$ - $p$ collision events. In Fig 4 the left and right peaks correspond to the EM incident and hadron incident events, respectively. The distribution was fitted with the MC template distributions generated for single gamma incident and single hadron incident events shown in the hatched histograms in Fig 4 in order to estimate efficiency and contamination for single gamma incident events. The MC template distributions were generated by the EPICS Monte Carlo simulator [18] with DPMJET3 for the interaction model. These procedure were carried out for each energy bin, and discrepancy of the "L90" distribution was taken into account as systematic errors. Luminosity measurement by FC gave a normalisation factor of data to the MC prediction with $6.1 \%$ uncertainty. Here inelastic cross section $\sigma_{\text {inel }}=71.5$ mb was assumed. Then incident position cuts were applied to select common pseudorapidity and azimuthal angle regions for two Arms. The large pseudorapidity and the small pseudorapidity regions were selected as $|\eta|>10.94$, and $8.81<|\eta|<8,99$ with $\Delta \phi=20$ degree, respectively. Finally both the Arm1 and Arm2 data were statistically combined.

Figure 5 shows the Arm1+Arm2 combined energy spectra of gamma rays produced in the two pseudorapidity regions from $\sqrt{s}=7 \mathrm{TeV} p$ - $p$ collisions compared with the 


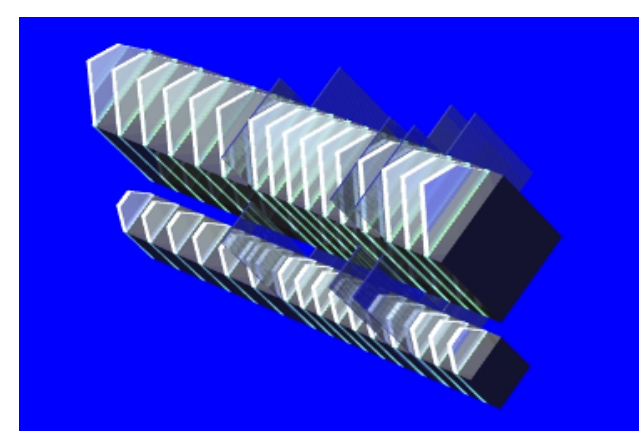

Figure 3. The LHCf detector Arm1(left) and Arm2(right).

MC prediction with various interaction models, SYBILL, DPMJET3, QGSJET-II, EPOS and PYTHIA [19]. The systematic errors shown in hatched boxes were mainly due to energy scale uncertainty and selection of EM showers. Uncertainty in overall normalisation of $6.1 \%$ due to luminosity measurement by FC is not shown here. One can see that none of the models perfectly reproduce the data. Among the models, DPMJET3 or PYTHIA show too hard in the high energy region. While these models show disagreement, the data points are "bracketed" by the models. It indicates that uncertainty estimated for current air shower simulations looks reasonable.

A sample of $p-p$ collisions at $0.9 \mathrm{TeV}$ was also analysed by the similar method. The $0.9 \mathrm{TeV}$ data sample was taken on 2, 3 and 27 May 2010 with typical luminosity with typical luminosity $L 4 \sim 12 \times 10^{27}$. Integrated luminosity corresponds to $0.3 \mathrm{nb}^{-1}$. Fig. 6 shows the energy spectra of gamma rays produced in the pseudorapidity regions $8.77<|\eta|<9.46$ and $|\eta|>10.15$ at $\sqrt{s}=0.9 \mathrm{TeV}$ $p$ - $p$ collisions [20]. Similar features are commonly seen both in the $7 \mathrm{TeV}$ and $0.9 \mathrm{TeV}$ energy data.

\section{$2.2 p_{T}$ spectra for the Type-I $\pi^{0}$ sample}

The $\pi^{0}$ sample provides more direct information of particle production at the very forward. So far the UA7 data at $\mathrm{S} p \bar{p} \mathrm{~S}(\sqrt{s}=630 \mathrm{GeV})$ is available for the $p_{T}$ spectra of very forward $\pi^{0}$ production [22]. The data sample for the $\pi^{0}$ analysis was taken on 15 and 16 May 2010 with typical luminosity $L=5 \sim 6 \times 10^{28}$. Integrated luminosity corresponds to $2.53 \mathrm{nb}^{-1}\left(1.90 \mathrm{nb}^{-1}\right)$ for Arm1 (Arm2). Analysis of calorimeter data was done with the similar methods as used in single gamma sample. In the reconstruction of $\pi^{0}$, each gamma-ray is reconstructed by one calorimenter ("Type-I" $\left.\pi^{0}\right)$. On the other hand, two gamma-rays from decay of a $\pi^{0}$ with more than $1.5 \mathrm{TeV}$ would be contained in a single calorimeter aperture ("Type-II" $\pi^{0}$ ). They could be also reconstructed their invariant masses thanks to fine granularity of position sensitive layers. Even though the

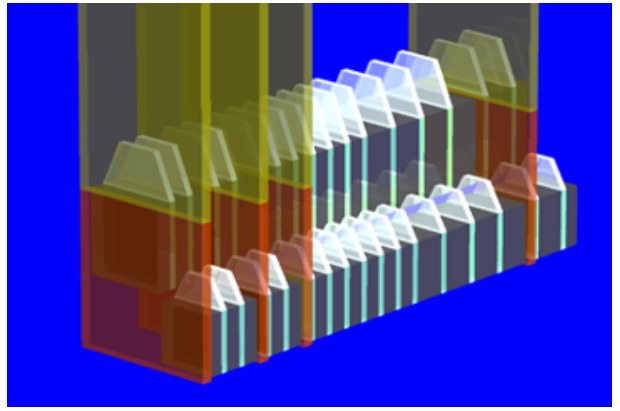

Type-II $\pi^{0}$ sample has resonablly good mass resolution, here we focus on Type-I $\pi^{0}$. In this analysis absolute energy scale was determined by the type-I $\pi^{0}$ masses to rescale the energy by $-8.1 \%$ (Arm1) and $-3.8 \%$ (Arm2). Figure 7 shows the invariant mass distributions for the Type-I and the Type-II $\pi^{0}$ samples. Mass resolution for Type-I and Type-II is $3.8 \%$ and about $8 \%$, respectively.

Figure 8 shows the $p_{T}$ spectra of Type-I $\pi^{0}$ 's reconstructed in the rapidity ranges $9.0<|y|<10.0$ at $7 \mathrm{TeV}$ $p$ - $p$ collisions [21]. Also shown the $p_{T}$ spectrum predicted by the various hadron interaction models, DPMJET3.04, QGSJET II-03, SIBYLL 2.1, EPOS 1.99 and PYTHIA 8.145. Similar to the single gamma sample, none of the models perfectly agrees, except EPOS shows better agreement with the data than other models. This different situation from the single gamma sample might be because Type-I $\pi^{0}$ 's and the $\pi^{0}$ 's for the origin of single $\gamma$ sample are not indentical. They originate in the different kienematical phase spaces (i.e. $P_{T}$ vs $E$ ).

Figure 9 shows average $\pi^{0} p_{T}$ for each rapidity region $y_{\text {lab }}$ obtained from $\sqrt{s}=7 \mathrm{TeV} p$ - $p$ collisions data at LHC together with UA7 $\sqrt{s}=630 \mathrm{GeV} p \bar{p}$ collision data taken at $\mathrm{S} p \bar{p} \mathrm{~S}$ [22]. Here $y_{\text {lab }}=y-y_{\text {beam }}$, where $y_{\text {beam }}=8.92$ for $\sqrt{s}=7 \mathrm{TeV}$ and $y_{\text {beam }}=6.50$ for $\sqrt{s}=630 \mathrm{GeV}$. The $\pi^{0} p_{T}$ data points obtained by LHCf covers much higher rapidity regions than those by $\mathrm{UA} 7$, and smoothly connected with each others and well alighn on the curves predicted by EPOS1.99 or QGSJETII. These two models show almost no collision energy depedence, while SYBILL shows slight dependence.

\section{Future prospects of the LHCf experiment}

Feedback of LHCf and other LHC data to air showers is also important issue. QGSJET-II and EPOS1.99 have been already updated to QGSJET II-4 and EPOS-LHC, respectively, to include new measurements done by all the LHC experiments. Impacts of this improvement on air shower 

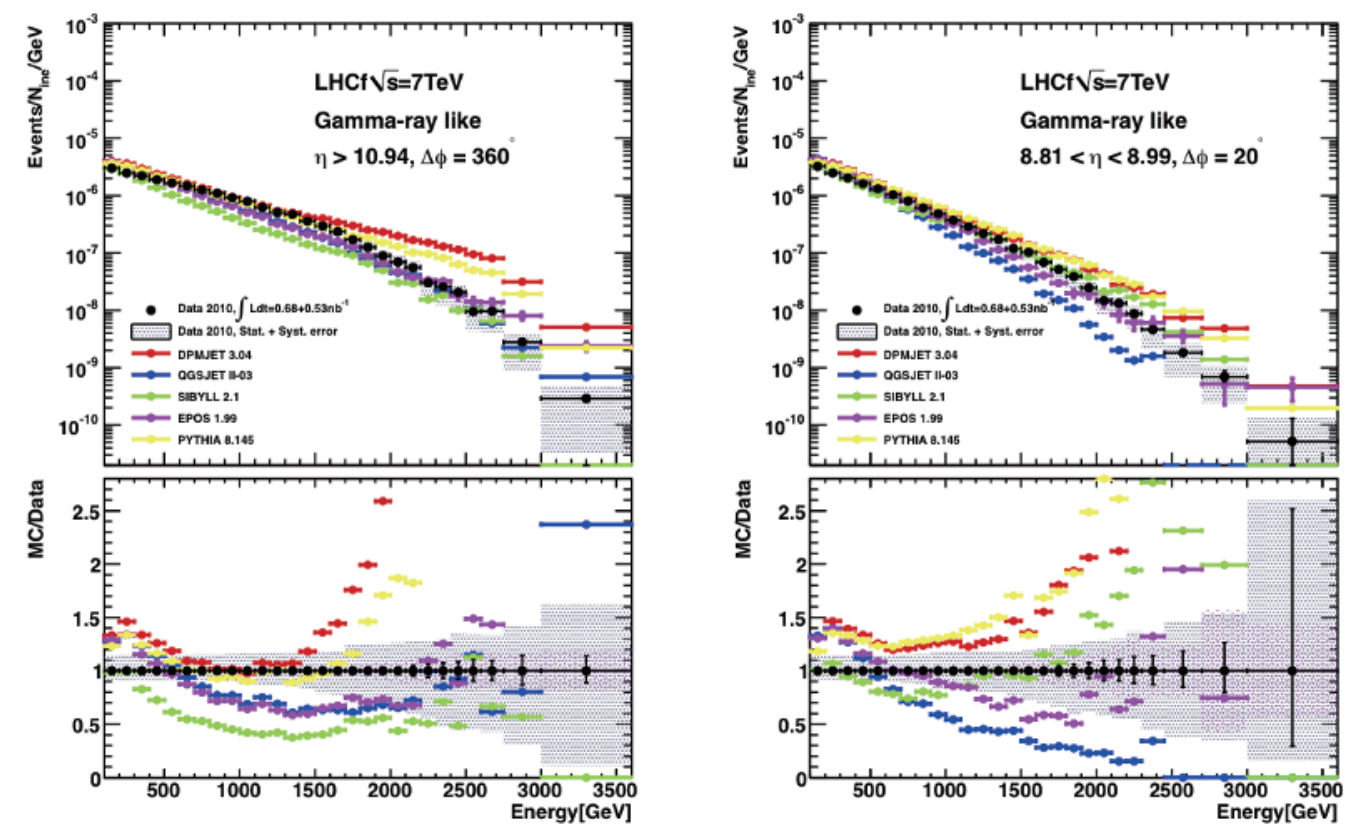

Figure 5. The energy spectra of single gamma-ray incident events in two pseudorapidity regions at $7 \mathrm{TeV}$ p-p collisions [19]. The black dots show the energy spectrum of single $\gamma$ sample in different rapidity ranges $\eta>10.94$ (left) and $8.81<\eta<8.99$ (right). Also shown the MC prediction by various hadron interaction models, SIBYLL 2.1 (green), EPOS 1.99 (purple), QGSJET II-03 (blue), PYTHIA 8.145 (yellow), and DPMJET3.04 (red). The top two and the bottom two panels show the event rate per collisions and the MC/Data ratios, respectively.
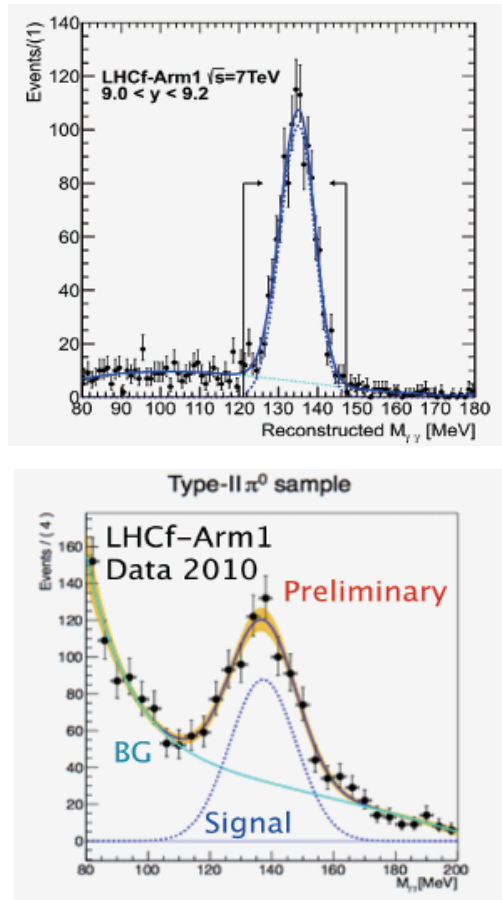

Figure 7. The invariant mass distributions for Type-I $\pi^{0}$ 's (top) and Type-II $\pi^{0}$,s (bottom).

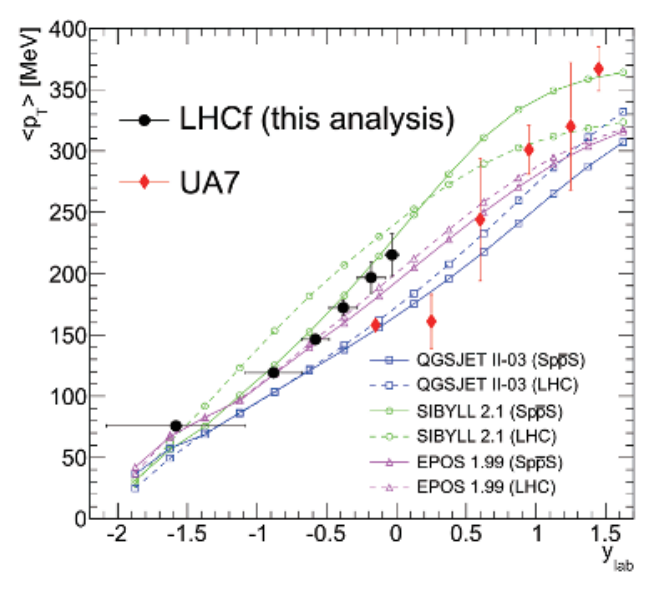

Figure 9. Average of $p_{T}$ spectra of Type-I $\pi^{0}$ 's for each rapidity $y_{\text {lab }}$ region [21]. Also shown the average $p_{T}$ as a fucntion of $y_{\text {lab }}$ by the various hadron interaction models, QGSJET II-03 (blue), SIBYLL 2.1 (green) and EPOS 1.99 (purple) for two different collision energies (LHC $\sqrt{s}=7 \mathrm{TeV}$ and $\mathrm{S} p \bar{p} \mathrm{~S} \sqrt{s}=630 \mathrm{GeV}$ ) The solid and dashed curves indicate LHC energy and $\mathrm{S} p \bar{p} \mathrm{~S}$ energy, respectively. 

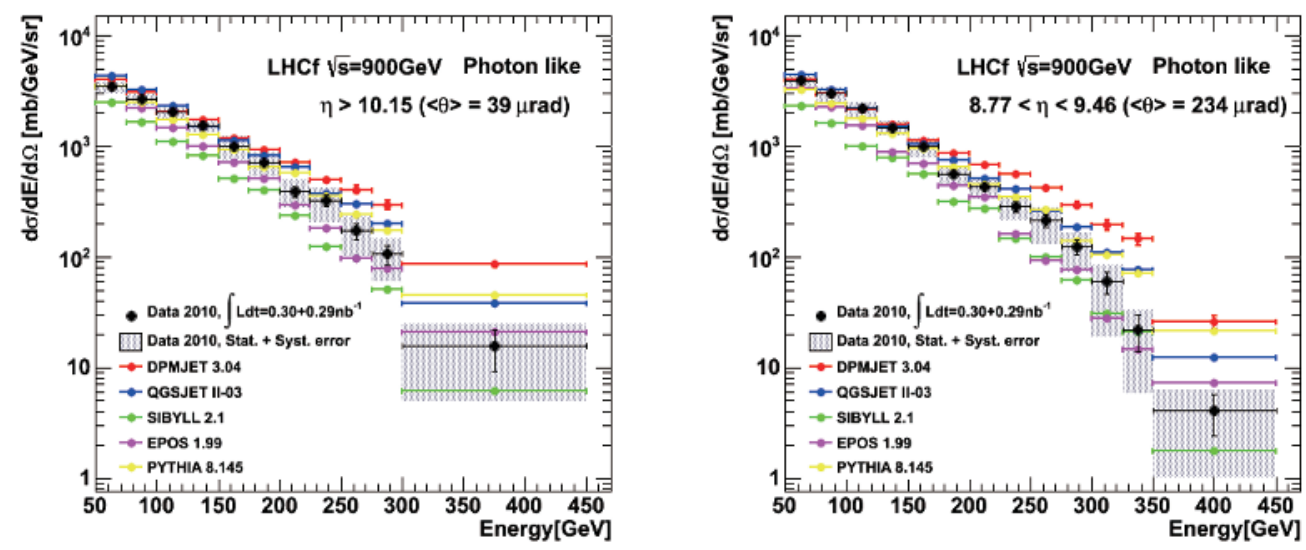

Figure 6. The energy spectra of single gamma-ray incident events in two pseudorapidity regions at $0.9 \mathrm{TeV}$ p-p collisions [20]. The black dots show the energy spectrum of single $\gamma$ sample in different rapidity ranges $\eta>10.15$ (left) and $8.77<\eta<9.46$ (right). Also shown the MC prediction by various hadron interaction models, SIBYLL 2.1 (green), EPOS 1.99 (purple), QGSJET II-03 (blue), PYTHIA 8.145 (yellow), and DPMJET3.04 (red). The top two and the bottom two panels show the differential cross section values and the MC/Data ratios, respectively.
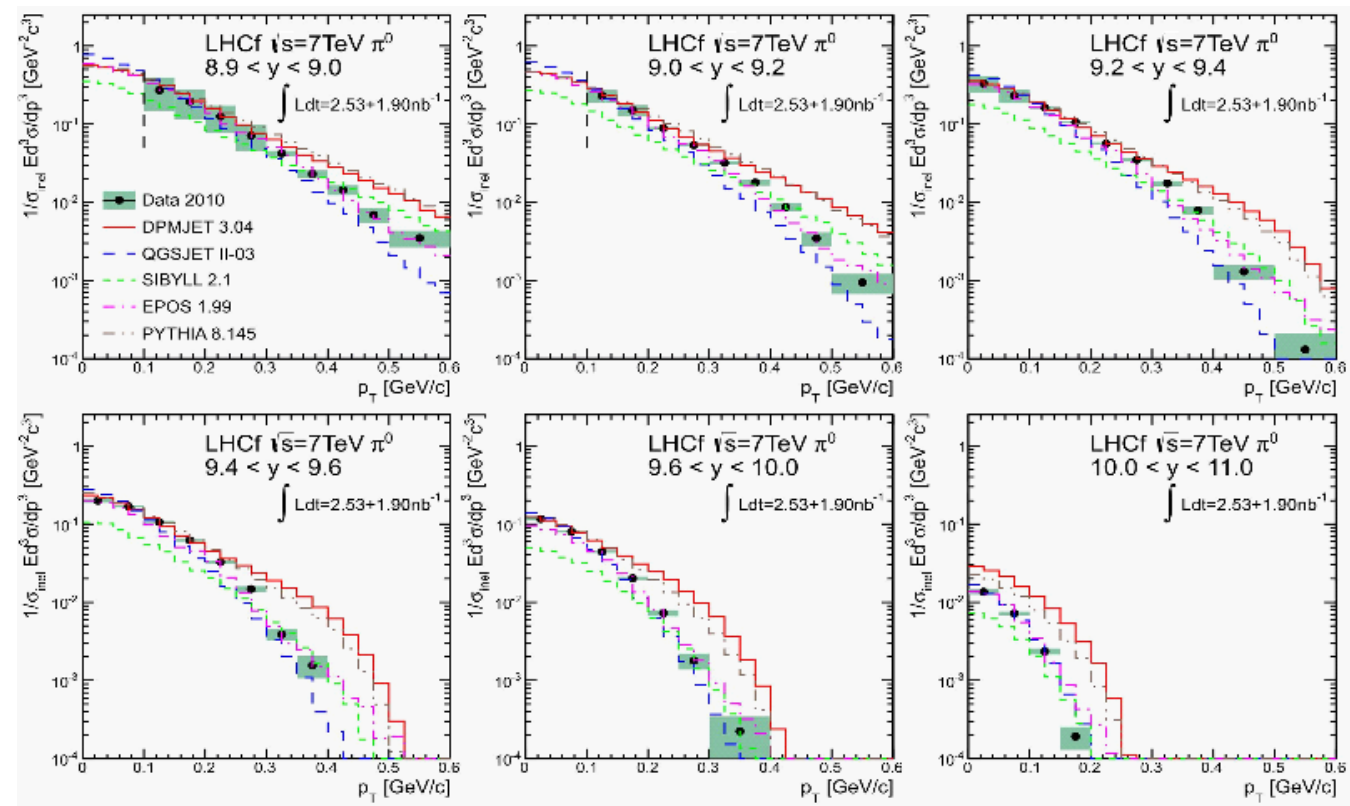

Figure 8. The $p_{T}$ spectra of Type-I $\pi^{0}$ 's reconstructed in six different rapidity ranges in $9.0<|y|<10.0$ at $7 \mathrm{TeV} p-p$ collisions [21]. Also shown the $p_{T}$ spectrum predicted by the various hadron interaction models, DPMJET3.04 (red solid), QGSJET II-03 (blue long dashed), SIBYLL 2.1 (green short dashed), EPOS 1.99 (purple dotted dashed) and PYTHIA 8.145 (gray dotted). 
measurement is discussed in [23]. Difference of Xmax prediction at the $10^{20} \mathrm{eV}$ region becomes improved and less energy-dependend.

There remains several parameters in cosmic ray interaction models need improvement as follows; inelasticity, nuclear effects and energy dependence. Inelasticity of collisions controls how large fraction of hadronic cascade component in air showers would survive at deeper site of atmosphere. It could largely modify the prediction of muon density at the ground in the current air shower simulations. It might relate to the ground muon excess anomaly problem recently reported by PAO [1] or KASCADE-GARNDE. Inelasticity can be checked by the neutron measurement at the very forward by LHCf. Figure 10 shows the MC expectation for energy distributions of very forward neutrons from $14 \mathrm{TeV} p$ - $p$ collisions detected by the LHCf detector. Because hadron interaction length of the LHCf detector (1.6 $\lambda$ ) is not enough to contain hadron shower, energy resolution for neutron measurement is expected to be about $30 \%$. But it is irrelevant to determine overall inelasticity from gamma / hadron ratio measurement. And even with such resolution we can recognize the difference of energy spectra predicted by various cosmic ray interaction models. Currently neutron analysis in LHCf $7 \mathrm{TeV} p$ - $p$ data is under progress. It should give an interesting impact on this issue.

Understanding energy dependence of very forward particle productions important to extraporate our knowledge on the intraction from th LHC energy to to $10^{20} \mathrm{eV}$. We have already learned more or less from the comparison between the single gamma spectra take at $0.9 \mathrm{TeV}$ and at $7 \mathrm{TeV}$. It would suggest pretty good scaling of Feynmann $x$ observed in this measurement. Futther verification will be done by future $13 \mathrm{TeV}$ collision data. Not only a new measurement at higher energy, but also new forward data at lower energy side should be important to understand energy dependence.

Cold nuclear effect in the forward region is also an important issue. Data taking of LHC p-Pb lead collisions at $\sqrt{s_{N N}}=5 \mathrm{TeV}$ was carried out in Jan/Feb 2013 by the LHCf Arm2 detector successfully. These data will verify nuclear effects used in cosmic ray interaction models, which affect energy spectra, multipicity, and $p_{T}$ of secondary particles. It is very useful to study impact parameter dependence of such very forward particle production to understand how nuclear effects modify secondary particle production. Collision centrariy can be detemined for $\mathrm{p}-\mathrm{A}$ collsiuons with the help of information from the ATLAS central detector. During this data taking successfull trigger exchange to scynchronize common data taking was carried out. Further collaborative analysis works with ATLAS is under going.

In order to understand collision energy dependence and A-dependence of cold nuclear effects in $\mathrm{p}$-A collisions, RHIC is interesting facility. The accelerator layout for the interaction region at RHIC is quite similar to that of LHC machine. A new zero degree measurement at RHIC is under discussion. In RHIC, there is also Zero Degree Calorimeter in the croatch of Y-shaped chamber similar to

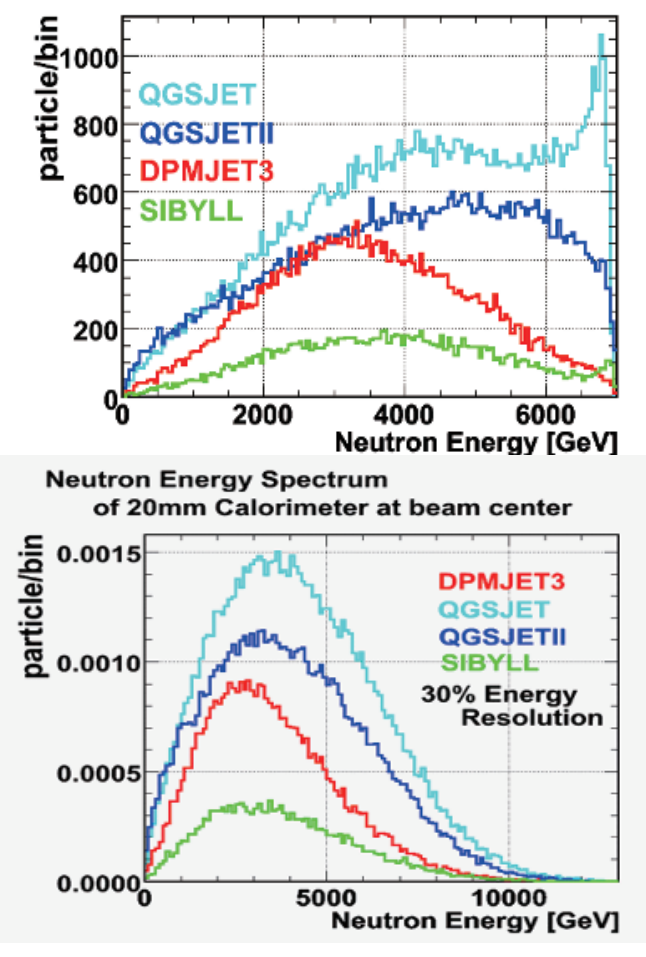

Figure 10. The expected energy distribution of the very forward neutrons detected by the LHCf detector from $14 \mathrm{TeV} p-p$ collisions calculated by the various interaction models such as QGSJET I (clear blue), QGSJET II-03 (blue), DPMJET3 (red), and SIBYLL 2.1 (green). The top and bottom plots show the energy distributions without and without the $30 \%$ energy resolution, respectively.

the LHC ZDC location. This location provides a $10 \mathrm{~cm}$ transverse space between two beam pipes. Since the LHC ZDC location is $140 \mathrm{~m}$ from the IP, the opening angle from $\pi^{0}$ of the energy lower than several hundred $\mathrm{GeV}$ is outside of the acceptance limited by the beampipe aperture as shown in Fig 11. However in the RHIC case, the distance between the collision point and the ZDC location is $20 \mathrm{~m}$ which is much closer than that in the LHC layout. Therefore acceptance becomes much larger so that reconstruction of $\pi^{0} \rightarrow 2 \gamma$ from $\sqrt{s}=500 \mathrm{GeV} p$ - $p$ collisions is possible. It provides direct comparision of $\pi^{0}$ production from the LHCf $7 \mathrm{TeV} p$ - $p$ collision data. Also RHIC has rich experience for various nuclear beams. Possible light ion-light ion or proton-light ion collisions can probe cosmic ray interactions for proton-nitrogen or Iron-nitrogen. Note that LHC has also such a capabiltiy for light ion collisions at UHECR energy in some future.

As originally planed, the LHCf detector will revisit LHC to take data of $13 \mathrm{TeV}$ collisions forseen in 2015. Refabrication of the LHCf detectors is now in progress to cope with high-radiation environment expected during $13 \mathrm{TeV} p$ - $p$ collisions. All the plastic scintillator layers and the plastic scintillation fibers are replaced by rad-hard GSO scintillator layers and fine segmented GSO scintillator bars, respectively [24]. The very forward data of 13 


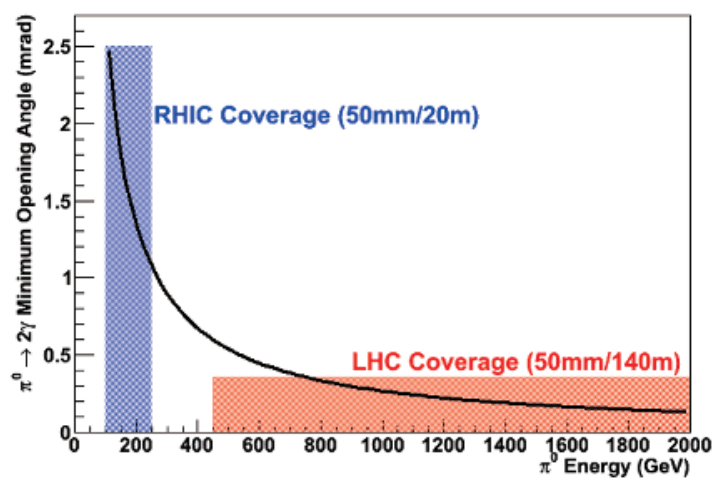

Figure 11. The opening angle as a fuction of $\pi^{0}$ energy. Also shown the possible acceptance for $\pi^{0} \rightarrow 2 \gamma$ at the RHIC ZDC site (blue) and the acceptance at the LHC ZDC site.

TeV $p$ - $p$ collisions will be an important next step to cosmic ray interaction. But also other opportunities, the data at lower collision energies and study nuclear effect by $\mathrm{p}$ A collisions, are essentially important to understand air shower phenomena.

\section{References}

[1] P. Abreu et al. [The Pierre-Auger collaboration], “ICRC2011, Beijing, Jul. 2011" arXiv:1107.4809

[2] Y. Tsunesada et al., [The Telscope Array collaboration], "ICRC2011, Beijing, Jul. 2011" arXiv:1111.2507

[3] K.Greisen, Phys. Rev. Lett, 16, 748 (1966).

[4] W.D.Apel, et al., "ICRC2011, Beijing, Jul. 2011", arXiv:1111.5436

[5] C. Meurer et al., [AUGER collaboration], Astrophys. Space. Sci. Trans. 7, 183 (2011).
[6] G.Thomson, J. Phys. Conf. Ser, 47, 248 (2006).

[7] E.J. Ahn et al., Phys. Rev. D80, 094003 (2009).

[8] S. Ostapchenko, Phys. Lett. 636, 40 (2006).

[9] S. Roesler, R. Engel and J. Ranft, Proc. of 27th Int. Cosmic Ray Conf. 2002, 439.

[10] T. Pierog and K. Werner, Phys. Rev. Lett. 101, 171101 (2008).

[11] G.Antchev et al. [The TOTEM collaboration], Euro. Phys. Lett. 98, 31002 (2012).

[12] V.Andreev et al. [The CMS collaboration], Euro. Phys. J. C67, 601 (2010).

[13] O.Adriani et al. [LHCf collaboration], JINST 3, S08006 (2008).

[14] T.Sako et al. [LHCf collaboration], Nucl. Instr. Meth. A578, 146 (2007).

[15] H.Menjo et al. [LHCf collaboration], Astropart. Phys. 34, 513 (2011).

[16] K.Taki. [LHCf collaboration], JINST 7(2012), T01003.

[17] T.Mase et al. [LHCf collaboration], Nucl. Instr. Meth. A671, 129 (2012).

[18] K.Kasahara, Proc. of 24th Int. Cosmic Ray Conf 1, 399 (1995)

[19] O.Adriani et al. [LHCf collaboration], Phys. Lett. B703, 128, (2011).

[20] O.Adriani et al. [LHCf collaboration], Phys. Lett. B715, 298 (2012).

[21] O.Adriani et al. [LHCf collaboration], Phys. Rev. D86, 092001 (2012).

[22] E. Paré et al. [UA7 collaboration], Phys. Lett. 242, 531 (1990).

[23] T.Pierog, "LHC data and extensive air shower", ISVHECRI2012, 10-15 Aug., 2012. Berlin.

[24] K.Kawade et al., JINST 6 T09004 (2011). 